\title{
Clinical Impact of Genomic Information in Pediatric Leukemia
}

\author{
Emilie Lalonde', Gerald Wertheim ${ }^{2}$ and Marilyn M. $\mathrm{Li}^{2,3 *}$ \\ ${ }^{1}$ Department of Genetics, Perelman School of Medicine, University of Pennsylvania, Philadelphia, PA, United States, \\ ${ }^{2}$ Department of Pathology and Laboratory Medicine, Perelman School of Medicine, University of Pennsylvania, Philadelphia, \\ PA, United States, ${ }^{3}$ Center for Childhood Cancer Research, The Children's Hospital of Philadelphia, Perelman School of \\ Medicine, University of Pennsylvania, Philadelphia, PA, United States
}

OPEN ACCESS

Edited by: Brenton Garrett Mar Dana-Farber Cancer Institute, United States

Reviewed by: Valentina Nardi, Partners HealthCare, United States

Frank Kuo,

Brigham and Women's Hospital, United States Marian Harris, Harvard University, United States

*Correspondence: Marilyn M. Li lim5@email.chop.edu

Specialty section: This article was submitted to Pediatric Hematology and Hematological Malignancies, a section of the journal Frontiers in Pediatrics

Received: 15 September 2017 Accepted: 24 November 2017 Published: 14 December 2017

Citation: Lalonde E, Wertheim G and Li MM (2017) Clinical Impact of Genomic Information in Pediatric Leukemia. Front. Pediatr. 5:263. doi: 10.3389/fped.2017.00263
Pediatric leukemia remains a significant contributor to childhood lethality rates. However, recent development of new technologies including next-generation sequencing (NGS) has increased our understanding of the biological and genetic underpinnings of leukemia, resulting in novel diagnostic and treatment paradigms. The most prevalent pediatric leukemias include B-cell acute lymphoblastic leukemia (B-ALL) and acute myeloid leukemia (AML). These leukemias are highly heterogeneous, both clinically and genetically. There are multiple genetic subgroups defined by the World Health Organization, each with distinct clinical management. Clinical laboratories have started adopting genomic testing strategies to include high-throughput sequencing assays which, together with conventional cytogenetic techniques, enable optimal patient care. This review summarizes genetic and genomic techniques used in clinical laboratories to support management of pediatric leukemia, highlighting technical, biological, and clinical advances. We illustrate clinical utilities of comprehensive genomic evaluation of leukemia genomes through clinical case examples, which includes the interrogations of hundreds of genes and multiple mutation mechanisms using NGS technologies. Finally, we provide a future perspective on clinical genomics and precision medicine.

Keywords: genomic profiling, pediatric leukemia, diagnosis, prognosis, therapy

\section{INTRODUCTION}

Leukemia accounts for $30 \%$ of all childhood malignancies in the United States $(1,2)$. Advances in the biological understanding of leukemogenesis and improved treatment options have significantly increased survival rates, with childhood mortality rates decreasing nearly fourfold from 1975 to 2016 (1-3). Pediatric leukemia is both clinically and genetically heterogeneous, and identifying personalized management schemes for every child with leukemia requires a thorough molecular investigation (4). Empowered by the development of next-generation sequencing (NGS) technologies, a large number of leukemia-associated genetic alterations have been elucidated in recent years. The wealth of basic research illustrating the genetic heterogeneity in pediatric leukemia has led to management changes in the workup of patients with leukemia and involves novel technologies. Many recent studies support the use of comprehensive genomic characterization of pediatric cancers in identifying potentially actionable mutations $(4,5)$.

This article focuses on the genomic evaluation of pediatric leukemia that may enable precision medicine. Biological and technical perspectives are considered in this review as we discuss molecular genetic strategies required for thorough analysis of these genetically heterogeneous malignancies. 
We discuss the strengths and weaknesses of various technologies required to inform clinical diagnosis, prognosis and management of pediatric leukemia, with an emphasis on the testing strategy employed at the Division of Genome Diagnostics from the Children's Hospital of Philadelphia (CHOP).

\section{MOLECULAR STRATEGIES FOR GENETIC TESTING IN LEUKEMIA}

The repertoire of clinically significant genomic mutations in hematological malignancies includes a wide variety of alterations. These include single-nucleotide variants (SNVs) leading to missense or nonsense amino acid changes; splice site substitutions affecting normal RNA processing; small deletions, duplications, insertions, or a combination of these commonly referred to as indels; copy number variations (CNVs); and large structural variations, disrupting the function of the genes involved or resulting in new fusion genes. Many of these alterations are critical for clinical diagnosis, prognosis, and therapy of patients with leukemia. Various molecular biology techniques are used clinically to detect these alterations. Chromosomal analysis, fluorescent in situ hybridization (FISH), and targeted Sanger sequencing have been the primary tools of detecting these alterations and remain part of the standard care (6). In recent years, high-throughput molecular technologies, including chromosomal microarray analysis (CMA) and NGS, have enhanced the capability to characterize critical genomic variations, and a combination of both classic and novel molecular technologies are used to assess clinically relevant mutations.

\section{Conventional and Molecular Cytogenetics}

Cancer cytogenetics started with the identification of the socalled Philadelphia chromosome, caused by a translocation between chromosomes 9 and 22, in the majority of patients with chronic myelocytic leukemia (CML) $(7,8)$. Numerous additional chromosomal rearrangements and copy number changes have since been identified. These abnormalities are either pathognomonic for specific hematologic malignancies, or convey prognostic and therapeutic implications. However, the genomic resolution is low (approximately $20 \mathrm{Mb}$ ), and viable, dividing cells must be obtained. FISH technology allows the detection of small (50-100 kb) deletions/duplications/amplifications and gene fusions, and permits the direct visualization of these alterations in interphase cells, yet is restricted to specific sequences of probes. As a complement to chromosomal analysis, CMA enables high-resolution detection of CNVs, allelic imbalance, and loss of heterozygosity (LOH) $(9,10)$, but cannot detect balanced structural changes, such as translocations or inversions. Despite the drawbacks of being manually intensive and not being scalable, karyotyping and FISH are still routinely performed for all diagnostic specimens as part of standard care.

\section{Polymerase Chain Reaction (PCR) and Sanger Sequencing}

Polymerase chain reaction followed by fragment analysis or Sanger sequencing can be used to detect clinically relevant alterations in leukemia, such as FLT3-ITD, B- and T-cell gene rearrangement analyses, or JAK2, NPM1, and many other gene mutations (11-15). Quantitative reverse transcription PCR (RT-qPCR) or digital droplet PCR have exquisite analytical sensitivity and may thus be used for detection of minimal residual diseases (MRDs) and for monitoring disease burden (e.g., $B C R-A B L 1$ fusion transcript levels in CML during tyrosine kinase inhibitor treatment) (16). As with FISH, however, these technologies are limited in the number of mutations they can evaluate at the same time, and are thus time consuming and costly.

\section{Next-Generation Sequencing}

As the cost of NGS decreases and the knowledge of mutational landscapes involved in leukemogenesis increases, NGS-based tests are quickly assuming critical roles in clinical cancer care. NGS is capable of detecting all forms of genomic alterations, and can scale from targeted panels (typically 50 to a few 100 genes), to whole-exome sequencing (WES) covering $1 \%$ of the genome, to whole-genome sequencing (WGS) (17). WES evaluates all coding sequences of the genome and WGS offers the most comprehensive mutational analysis by sequencing the whole genome (Table 1). However, both WES and WGS are not routinely used in clinical laboratories due to the cost, long turnaround time, and extensive efforts required for data analysis at present time $(18,19)$. As such, clinical laboratories have begun to adopt NGS panel assays restricted to detecting mutations in specific genes known to be essential in leukemia. Compared to traditional Sanger sequencing or FISH assays, targeted NGS panel tests are far more robust, cost-effective, and can provide more comprehensive genomic information in a shorter time-frame (19-21). For these targeted assays, different strategies have been developed to capture the regions of interest from both DNA and RNA, including amplicon sequencing from multiplex PCR, hybrid capture using magnetic beads, and anchored multiplex PCR. Whereas multiplex PCR may be best suited for small panel sizes and has lower input requirements, hybrid capture can target hundreds of genes and results in more even coverage which enables copy number analysis. Anchored multiplex PCR is best suited to identify structural rearrangements leading to known or novel fusion genes. However, it is expected that broader assays such as exome, genome, and transcriptome sequencing, will eventually replace panel-based tests as costs continue to decrease and analytical methods improve.

In our clinical laboratory, we have developed a NGS-based comprehensive hematological cancer panel to profile genomic alterations of all leukemia patients at diagnosis and at relapse. This panel is designed to interrogate SNVs, indels, CNVs/LOH in more than 100 genes, and both known and novel fusions associated with 106 major fusion partners. Variants identified from these assays are put into clinical context using the standards and guidelines for somatic variant interpretation and reporting defined by AMP, ASCO, CAP, and ACMG, highlighting clinically actionable variants (22). Although this thorough approach has required extensive investment in infrastructure, equipment, information technology, data storage, and skilled personnel, the enhanced analysis has clearly impacted care of pediatric leukemia patients. 
TABLE 1 | Comparison of genomic technologies commonly used in clinical laboratories for leukemia profiling.

\begin{tabular}{|c|c|c|c|c|c|}
\hline & Cytogenetics & $\begin{array}{l}\text { Fluorescent in situ } \\
\text { hybridization }\end{array}$ & $\begin{array}{l}\text { Chromosomal } \\
\text { microarray analysis }\end{array}$ & $\begin{array}{l}\text { Sanger } \\
\text { sequencing }\end{array}$ & $\begin{array}{l}\text { Next-generation } \\
\text { sequencing }\end{array}$ \\
\hline Resolution & $10-20 \mathrm{Mb}$ & $50-100 \mathrm{~kb}$ & $1-100 \mathrm{~kb}$ & $1 \mathrm{bp}$ & $1 \mathrm{bp}$ \\
\hline Sample type & $\begin{array}{l}\text { Fresh tissue: PB, BM, } \\
\text { tumor }\end{array}$ & $\begin{array}{l}\text { PB, BM, fresh, FF, } \\
\text { FFPE, etc. }\end{array}$ & $\begin{array}{l}\text { PB, BM, fresh, FF, FFPE, } \\
\text { etc. }\end{array}$ & $\begin{array}{l}\text { PB, BM, fresh, FF, FFPE, } \\
\text { etc. }\end{array}$ & PB, BM, fresh, FF, FFPE, etc. \\
\hline Aberration detection & $\begin{array}{l}\text { Del/Dup/Amp, insertion, } \\
\text { translocation }\end{array}$ & $\begin{array}{l}\text { Del/Dup/Amp, } \\
\text { translocation }\end{array}$ & Del/Dup/Amp & SNVs, indels & $\begin{array}{l}\text { Del/Dup/Amp, translocation, } \\
\text { SNV, indel, fusions, SV }\end{array}$ \\
\hline $\begin{array}{l}\text { Loss of } \\
\text { heterozygosity }\end{array}$ & No & No & Yes $^{\mathrm{a}}$ & No & Yes \\
\hline $\begin{array}{l}\text { Qualitative or } \\
\text { quantitative }\end{array}$ & $\begin{array}{l}\text { Qualitative with low } \\
\text { sensitivity }\end{array}$ & Quantitative & Semi quantitative & $\begin{array}{l}\text { Qualitative with relatively } \\
\text { low sensitivity }\end{array}$ & Quantitative with high sensitivity \\
\hline Genomic coverage & Whole genome & Targeted & Whole genome & Targeted & Targeted/whole genome \\
\hline Scalability & No & No & Yes & No & Yes \\
\hline
\end{tabular}

anly arrays containing SNPS.

PB, peripheral blood; BM, bone marrow; FF, fresh frozen; FFPE, formalin fixed paraffin imbedded; del, deletion; dup, duplication; Amp, amplification; SV, structural variation; UPD, uniparental disomy; SNVs, single nucleotide variants.

\section{CLINICAL UTILITY OF GENOMIC ANALYSIS IN LEUKEMIA}

\section{Genomic-Guided Management in Acute Lymphoblastic Leukemia (ALL)}

Acute lymphoblastic leukemia is the most common childhood malignancy accounting for $75-80 \%$ of pediatric leukemia and $26 \%$ of cancer diagnosed before 14 years of age $(23,24)$. Genetic rearrangements and CNVs characterize 10 different B-cell ALL subgroups as defined by the World Health Organization (WHO), including two provisional groups, each with distinct clinical considerations (25). Recent guidelines from the College of American Pathologists and the American Society of Hematology echo the WHO scheme in terms of the importance of genetic testing in acute leukemias (26). These recommendations include testing for common genomic structural changes and recurrent point mutations commonly seen in pediatric ALL and acute myeloid leukemia (AML).

The first provisional WHO subgroup, Ph-like B-cell acute lymphoblastic leukemia (B-ALL), is defined by a similar gene expression profile to B-ALL with the Philadelphia chromosome yet lacks a $B C R-A B L 1$ fusion (27). Approximately $10 \%$ of pediatric $\mathrm{B}-\mathrm{ALL}$ may be classified as $\mathrm{Ph}$-like ALL, and show poor response to induction chemotherapy (28). These leukemias harbor gene fusions involving tyrosine kinases including $A B L 1$ (not associated with BCR), ABL2, PDGFRB, NTRK3, TYK2, CSF1R, and $J A K 2$, rearrangements involving cytokine receptor-like factor 2 (CRLF2), or alterations associated with erythropoietin receptor gene. These mutations lead to activation of growth promoting kinases or of cytokine signaling pathways (29). ALL with CRLF2 rearrangements often found in combination with JAK mutations, resulting in activation of JAK-STAT pathways $(30,31)$. These distinct alterations may render these leukemias sensitive to tyrosine kinase inhibitors such as dasatinib, JAK inhibitors such as ruxolitinib, or other small molecule inhibitors (29). Current clinical trials, such as COG AALL1521, incorporate mutational testing and small molecular inhibitors in their protocols (https://clinicaltrials.gov/, e.g., NCT02723994). It is, therefore, imperative to include $\mathrm{Ph}$-like alterations in the molecular testing of B-ALL. Additionally, many of the kinases involved in fusions have multiple fusion partners with distinct downstream biological effects, requiring testing modalities capable of detecting multiple known and even novel fusion partners.

The NGS testing repertoire in our laboratory incorporates novel fusion kinases involved in Ph-like B-ALL. We recently performed testing on an 11-year-old patient with relapsed B-ALL. The patient had previously tested negative by conventional technologies; however, we identified a PAX5-JAK2 fusion (Figure 1A) along with biallelic loss of TP53 function. PAX5 is a master regulator of $\mathrm{B}$-cell development, and the chimeric protein maintains both the PAX5 DNA-binding activity and the JAK2 kinase activity (32). Both loci are promiscuous in translocation partners, as JAK2 can include up to 14 different $5^{\prime}$ partners in Ph-like B-ALL (30), and PAX5 has at least 12 fusion partners (33). The PAX5-JAK2 fusion has previously been identified in $\mathrm{Ph}$-like $\mathrm{B}-\mathrm{ALL}$, and the chimeric protein has shown to be sensitive to JAK2 inhibitors by in vitro studies (32). The identification of the PAX5-JAK2 fusion indicates that the patient has a Ph-like ALL with poor prognosis and opens the door for possible therapy with JAK inhibitors.

The other provisional B-ALL subtype is characterized by intrachromosomal amplification of chromosome 21 (i.e., iAMP21) caused by breakage-fusion-bridge cycles followed by chromothripsis or other complex rearrangements $(25,34)$. The entity is defined as at least three copies of the RUNX1 gene on one chromosome 21, although the region of amplification is often larger, and may be associated with subtelomeric deletions of chromosome $21(31,34)$. It is mostly found in older children and is also associated with very poor prognosis with standard treatment protocols (34). Nevertheless, multiple clinical trials have demonstrated that more intensive chemotherapy regimens geared toward high-risk patients are effective in these patients, and counteract the adverse prognostic impact of iAMP21 $(35,36)$. 


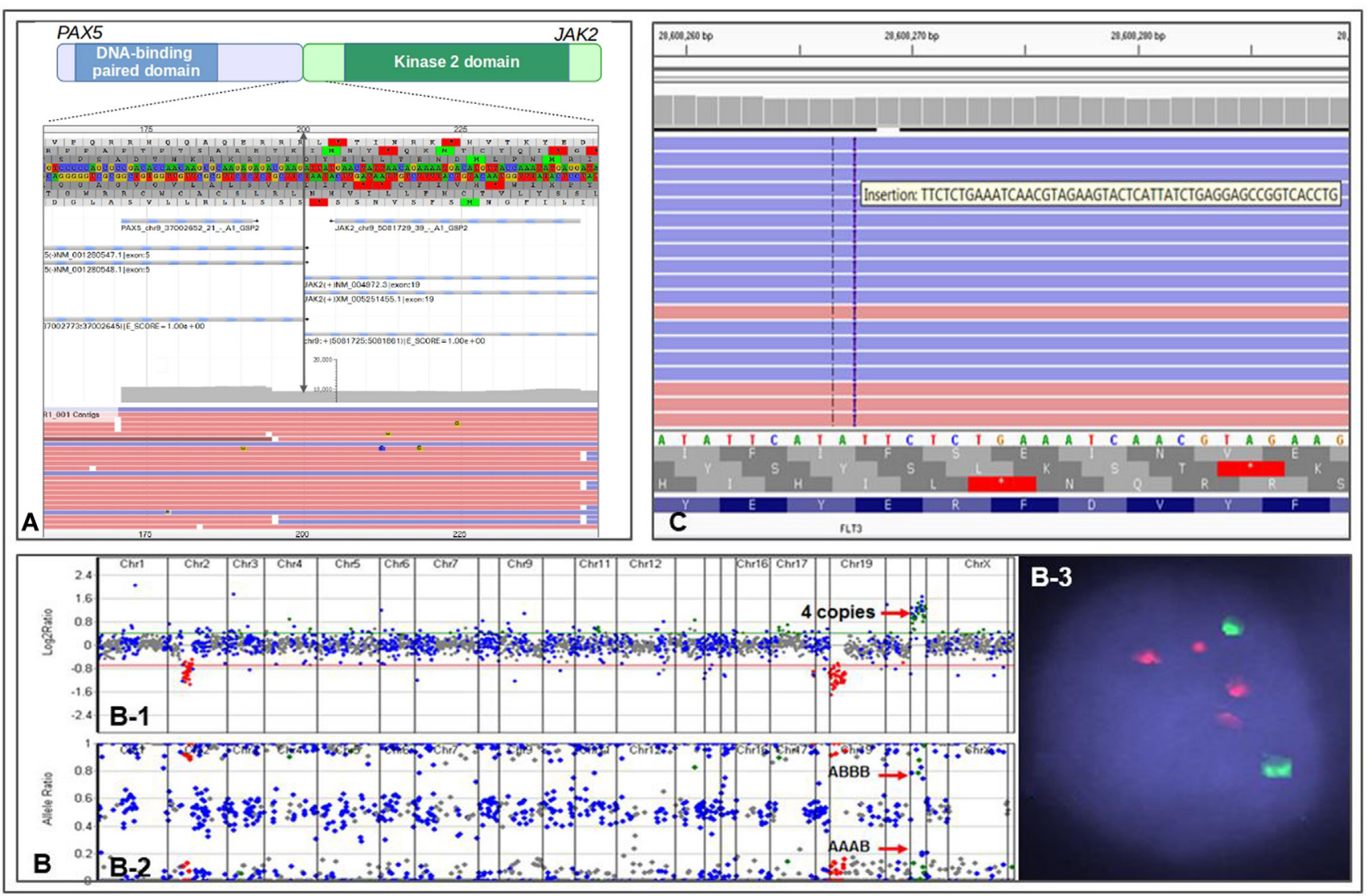

FIGURE 1 | Representations of different genomic alterations identified by CHOP Comprehensive Hematological Cancer Panel. (A) a PAX2-JAK2 fusion detected in an 11-month-old patient with residual/recurrent B-cell lymphoblastic leukemia. Double arrowed line indicates exon 5 of PAX2 is fused to exon 19 of JAK2, gray area indicates reading depth, red and blue horizontal bars are representative reads. (B) Copy number analysis using next-generation sequencing data from a patient with iAMP ALL. (B-1) Copy number variations analysis based on reading depth; red arrow indicates four copies of RUNX1 genomic region. (B-2) B-allele frequency analysis demonstrating SNP separation due to triplication of one allele; red arrows indicate genotype information of AAAB and ABBB (the genotype would be AABB if it were duplication of both allele). (B-3) FISH showing two ETV6 signals (green) and 4 RUNX1 signals (red). (C) IGV view showing a FLT3 ITD in an 11-year-old patient with acute myeloid leukemia.

One of the major benefits of using a NGS-based genetic testing strategy is the ability to detect multiple types of aberrations in a single assay. Indeed, we recently identified a case of iAMP21 ALL using our Comprehensive Hematological Cancer Panel. Our NGS analysis software uses sequencing depth and common SNPs in the targeted regions to assess copy number status and genotype information at the same time. We identified a patient with B-ALL who showed four RUNX1 signals by interphase FISH. This pattern could represent two signals on each chromosome 21 , or at least three signals on a single chromosome, the latter of which indicates an iAMP21. NGS analysis showed four copies of RUNX1 gene and a clear "AAAB" and "ABBB" heterozygous SNP pattern in the RUNX1 chromosome region, demonstrating an iAMP21 (Figure 1B). The patient was immediately placed on an appropriate high-risk chemotherapy regimen.

\section{Genomic-Guided Prognostication in AML}

Acute myeloid leukemia is an aggressive leukemia, with a cure rate of only approximately $60 \%$ for pediatric patients (37). Patients were historically classified into distinct subtypes of AML based on cell morphology and cytochemistry, yet are currently defined mainly by genetic abnormalities $(38,39)$. In 2001, the WHO classification of the myeloid neoplasms first recognized three subtypes of AML with recurrent genetic abnormalities (39). In 2008, the WHO classification included nine genomic alteration-based AML subtypes (40). The 2016 updates recognizes 11 genetically defined AML subtypes (25), highlighting the clinical significance of genomic testing in the care of AML patients.

A recent experience at $\mathrm{CHOP}$ illustrates well the importance of integrated genomic workup for AML. An 11-year-old patient with cytogenetically normal AML (CN-AML) was referred for further genetic testing for risk stratification. The Comprehensive Hematological Cancer Panel identified a FLT3 internal tandem duplication (FLT3 ITD) (Figure 1C) along with two other somatic variants, IDH2 p.R140Q and GATA2 p.W10*. FLT3 ITD is often missed by NGS-based assays due to the size of the duplications. However, we have designed our panel and bioinformatics analysis pipeline to enable the detection of this mutation. The FLT3 
variant changes the patient's prognosis from intermediate (based on CN-AML) to poor $(25,41-43)$. Moreover, recent clinical trials suggest adding FLT3 inhibitor to frontline chemotherapy in FLT3-mutated AML confers a survival benefit (44). The FLT3 ITD present in this patient qualifies her for clinical trials investigating FLT3 ITD targeted therapies.

\section{Genomic-Guided Diagnosis: Identifying RAS-Pathway Mutations in JMML}

Juvenile myelomonocytic leukemia (JMML) is a rare type of leukemia found mainly in children less than 2 years of age (45). The prognosis of JMML is poor, and without treatment it is most often fatal within 5 years of life. Hematopoietic stem cell transplant is currently the only curative approach for JMML. Since both the clinical presentation and the morphologic features of JMML can resemble reactive processes, molecular studies are usually required for diagnosis. Most individuals with JMML have mutations in the RAS pathway, particularly PTPN11, KRAS, $N R A S, C B L$, or NF1. Some mutations in the RAS-MAPK pathway genes can occur in the germline and these patients are at risk for JMML-like myeloproliferations. JMML patients without RAS mutations may have mutations in ASXL1, SETBP1, RUNX1, JAK3, and $S H 2 B 3(46,47)$, all of which have also been observed as secondary mutations in RAS-mediated JMML (46). In the 2016 WHO classification, the RAS-pathway mutations are among the diagnostic criteria for JMML (25).

Our Comprehensive Hematologic Cancer Panel includes the RAS-pathway genes and allows for rapid diagnosis of JMML. This is illustrated by the case of a 19-month-old boy with splenomegaly and WBC of 50.7 million per microliter with high count of monocytes, an absolute monocytosis, and thrombocytopenia. Blasts in both the peripheral blood and bone marrow were $<20 \%$. A rapid FISH analysis was negative for a $B C R-A B L 1$ translocation. The NGS panel was completed within 5 days of sample acquisition, and identified a missense mutation in PTPN11 (c.181G $>$ dT, p.Asp61Tyr). The genomic information helped us make the diagnosis within one week which allowed physicians to construct appropriate therapeutic strategies in a timely manner. Sanger sequencing on cultured and direct skin biopsies did not detect the variant, confirming the somatic origin.

\section{Genomic-Guided Disease Monitoring}

The ability to detect a large variety of mutations allows the potential for tracking disease burden and relapse through molecular assays. MRD detection can be used to assess therapeutic response and is invaluable for patient management $(14,48-50)$. Historically, MRD tracking is done by using flow cytometry or by RT-qPCR assays to detect specific aberrations such as $B C R-A B L 1$ fusion (48). In CML, an International Scale has been established to allow standardization and comparison of results from RT-qPCR BCR$A B L 1$ assays from different laboratories, and validated clinical endpoints have been established $(51,52)$. With the discovery of additional recurrent genetic mutations in hematological malignancies, new RT-qPCR assays have been developed to provide molecular tracking for MRD in a larger proportion of patients (53). This approach is particularly useful for patients undergoing targeted therapy.

Genomic analysis on a diagnostic bone marrow from an 11-year-old boy with very high-risk ALL and induction failure showed a novel GOLGA5-JAK2 fusion, indicating a Ph-like

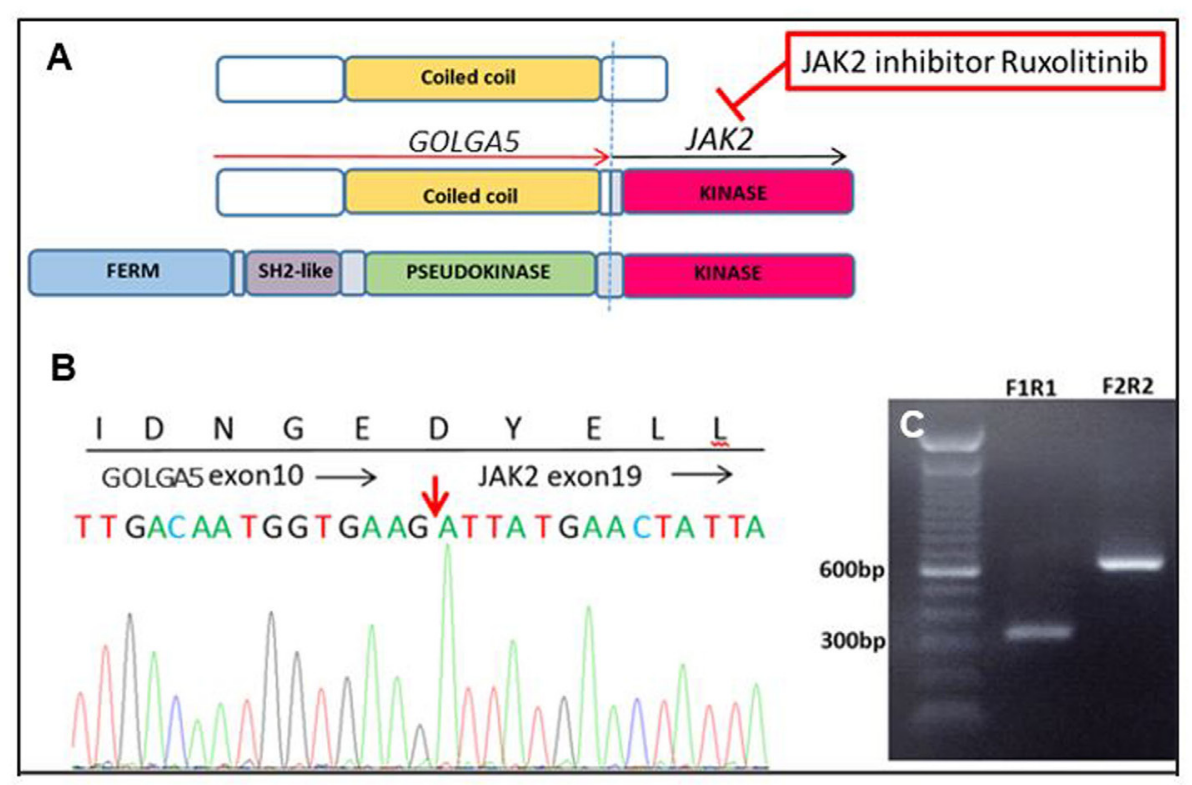

FIGURE 2 | A novel GOLGA5-JAK2 fusion was identified in an 11-year-old boy with very high-risk acute lymphoblastic leukemia. (A) Schematic representation of protein domains of the GOLGA5-JAK2 fusion protein. (B) Sanger confirmation of the GOLGA5-JAK2 fusion. The red arrow indicates the breakpoint of the fusion transcript. (C) Gel electrophoresis of nested polymerase chain reaction (PCR) products from the diagnostic bone marrow sample; F1R1 - PCR product of inner forward and reverse primers; F2R2 - PCR product of outer forward and reverse primers. 
B-ALL (Figure 2). Upon detection of the novel fusion, JAK inhibitor ruxolitinib was added to the therapy and a custom RT-qPCR assay was designed specifically for this patient to monitor the JAK2 fusion transcript levels during therapy. A nearly two-log reduction in the amount of JAK2 fusion transcripts was observed one month after the ruxolitinib treatment initiation, and the fusion transcripts were undetectable after 5 months of treatment. The patient was then enrolled in a clinical trial to receive CAR-T immunotherapy followed by matched sibling donor bone marrow transplant (BMT) and has been in remission ever since.

\section{SUMMARY AND PERSPECTIVES}

The technical advances in genomic research and their clinical applications have resulted in tremendous clinical improvements within the last decade. In hematological cancers, in particular, molecular profiling is used to diagnose disease, stratify risk, guide therapy, and monitor treatment responses with increasing accuracy (54). Channeling the power of artificial intelligence, several large initiatives have begun acquiring, storing, and retrospectively mining genomic and clinical information to inform treatment decisions for new patients (48-50). This knowledge bank approach calculates an individual patient's risk profile for various treatment options, based on observations from prior patients. Gerstrung and colleagues recently illustrated that individually tailored management decisions based on this approach could decrease the number of hematopoietic cell transplants in AML patients by $20-25 \%$ while maintaining the same overall survival rates (51).

Targeted NGS panels currently have a clinical advantage over exome and genome sequencing, due to faster turnaround times and higher sequencing depths resulting in higher analytical sensitivity and specificity (52). However, using targeted panels introduces significant logistical issues, in particular the need to

\section{REFERENCES}

1. Linet MS, Ries LA, Smith MA, Tarone RE, Devesa SS. Cancer surveillance series: recent trends in childhood cancer incidence and mortality in the United States. J Natl Cancer Inst (1999) 91(12):1051-8. doi:10.1093/jnci/91. 12.1051

2. Howlader N, Noone AM, Krapcho M, Miller D, Bishop K, Altekruse SF, et al. SEER Cancer Statistics Review, 1975-2013. National Cancer Institute (2016). Available from: https://seer.cancer.gov/csr/1975_2013/, based on November 2015 SEER data submission, posted to the SEER web site.

3. Wang $\mathrm{Y}$, Huang J, Rong $\mathrm{L}$, Wu $\mathrm{P}$, Kang $\mathrm{M}$, Zhang $\mathrm{X}$, et al. Impact of age on the survival of pediatric leukemia: an analysis of 15083 children in the SEER database. Oncotarget (2016) 7(50):83767-74. doi:10.18632/oncotarget. 11765

4. Mody RJ, Prensner JR, Everett J, Williams Parsons D, Chinnaiyan AM. Precision medicine in pediatric oncology: lessons learned and next steps. Pediatr Blood Cancer (2016) 64(3):e26288. doi:10.1002/pbc.26288

5. Rampal R, Levine RL. Leveraging cancer genome information in hematologic malignancies. JClin Oncol (2013) 31(15):1885-92. doi:10.1200/ JCO.2013.48.7447

6. Mikhail FM, Heerema NA, Rao KW, Burnside RD, Cherry AM, Cooley LD. Section E6.1-6.4 of the ACMG technical standards and guidelines: chromosome studies of neoplastic blood and bone marrow-acquired chromosomal abnormalities. Genet Med (2016) 18(6):635-42. doi:10.1038/gim.2016.50 re-design, re-optimize, and re-validate panels as new targets are discovered and become clinically relevant, imposing a burden on clinical laboratories wishing to update panel content on a regular basis. Targeted panels are also at risk of missing rare novel genomic alterations that are not included in a specific panel and are not suitable for many structural variations due to its reduced complexity. Given the decreasing cost and continued evolution of sequencing technologies and analytical methodologies, WGS, with its ability to identify SNVs, indels, CNVs/LOH, and structural variants simultaneously, and whole transcriptome sequencing that evaluates gene expression profiles, may soon supersede targeted panels $(29,53)$. Yet even WGS has limitations in that $8 \%$ of the genome cannot be sequenced due to repetitive sequences (55). Novel strategies such as long-read, single-molecule sequencing (e.g., PacBio, Oxford Nanopore) can fill some of these coverage gaps. These methods also do not require target amplification thereby reducing read strand bias (55-59). Linked read sequencing (10× Genomics), may also capture repetitive sequence regions of the genome, and enable strand phasing by using a barcoded, microfluidics approach (60). Development of these and other new technologies along with more sophisticated bioinformatics tools will permit an even more complete clinical representation of the molecular alterations in leukemia and offer patients exquisitely precise diagnosis, prognosis, and therapeutic opportunities.

\section{AUTHOR CONTRIBUTIONS}

EL, GW, and ML wrote, edited, and approved the final manuscript.

\section{FUNDING}

This study was supported in part by the Department of Pathology and Laboratory Medicine, Children's Hospital of Philadelphia, University of Pennsylvania, Perelman School of Medicine.

7. Rowley JD. A new consistent chromosomal abnormality in chronic myelogenous leukaemia identified by Quinacrine fluorescence and Giemsa staining. Nature (1973) 243(5405):290-3. doi:10.1038/243290a0

8. Nowell PC, Hungerford DA. A minute chromosome in human chronic granulocytic leukemia. Science (1960) 132:1497.

9. Surrey LF, Luo M, Chang F, Li MM. The genomic era of clinical oncology: integrated genomic analysis for precision cancer care. Cytogenet Genome Res (2016) 150(3-4):162-75. doi:10.1159/000454655

10. Li MM, Monzon FA, Biegel JA, Jobanputra V, Laffin JJ, Levy B, et al. A multicenter, cross-platform clinical validation study of cancer cytogenomic arrays. Cancer Genet (2015) 208(11):525-36. doi:10.1016/j.cancergen.2015. 08.002

11. Kottaridis PD, Gale RE, Langabeer SE, Frew ME, Bowen DT, Linch DC. Studies of FLT3 mutations in paired presentation and relapse samples from patients with acute myeloid leukemia: implications for the role of FLT3 mutations in leukemogenesis, minimal residual disease detection, and possible therapy with FLT3 inhibitors. Blood (2002) 100(7):2393-8. doi:10.1182/ blood-2002-02-0420

12. Pongers-Willemse MJ, Seriu T, Stolz F, d’Aniello E, Gameiro P, Pisa P, et al. Primers and protocols for standardized detection of minimal residual disease in acute lymphoblastic leukemia using immunoglobulin and $\mathrm{T}$ cell receptor gene rearrangements and TAL1 deletions as PCR targets Report of the BIOMED-1 CONCERTED ACTION: investigation of minimal residual disease in acute leukemia. Leukemia (1999) 13(1):110-8. 
13. Matsumura T, Kami M, Saito T, Sakamaki H, Hirai H. Prognostic value of minimal residual disease in acute lymphoblastic leukaemia in childhood. Lancet (1999) 353(9154):752. doi:10.1016/S0140-6736(05)76119-5

14. Ramasamy I, Brisco M, Morley A. Improved PCR method for detecting monoclonal immunoglobulin heavy chain rearrangement in B cell neoplasms. J Clin Pathol (1992) 45(9):770-5. doi:10.1136/jcp.45.9.770

15. Sanger F, Nicklen S, Coulson AR. DNA sequencing with chain-terminating inhibitors. Proc Natl Acad Sci U S A (1977) 74(12):5463-7. doi:10.1073/ pnas.74.12.5463

16. Mencia-Trinchant N, Hu Y, Alas MA, Ali F, Wouters BJ, Lee S, et al. Minimal residual disease monitoring of acute myeloid leukemia by massively multiplex digital PCR in patients with NPM1 mutations. J Mol Diagn (2017) 19(4):537-48. doi:10.1016/j.jmoldx.2017.03.005

17. MacConaill LE. Existing and emerging technologies for tumor genomic profiling. J Clin Oncol (2013) 31(15):1815-24. doi:10.1200/JCO.2012.46.5948

18. Rehm HL, Hynes E, Funke BH. The changing landscape of molecular diagnostic testing: implications for academic medical centers. J Pers Med (2016) 6(1):8. doi:10.3390/jpm6010008

19. Rehm HL. Evolving health care through personal genomics. Nat Rev Genet (2017) 18(4):259-67. doi:10.1038/nrg.2016.162

20. Liu L, Li Y, Li S, Hu N, He Y, Pong R, et al. Comparison of next-generation sequencing systems. J Biomed Biotechnol (2012) 2012:251364. doi:10.1155/ 2012/251364

21. Shendure J, Ji H. Next-generation DNA sequencing. Nat Biotechnol (2008) 26(10):1135-45. doi:10.1038/nbt1486

22. Li MM, Datto M, Duncavage EJ, Kulkarni S, Lindeman NI, Roy S, et al. Standards and guidelines for the interpretation and reporting of sequence variants in cancer. J Mol Diagn (2017) 19(1):4-23. doi:10.1016/j.jmoldx. 2016.10.002

23. Ward E, DeSantis C, Robbins A, Kohler B, Jemal A. Childhood and adolescent cancer statistics, 2014. CA Cancer J Clin (2014) 64(2):83-103. doi:10.3322/ caac. 21219

24. American Cancer Society. What Are the Key Statistics for Childhood Leukemia? (2016). Available from: https://www.cancer.org/cancer/leukemiain-children/about/key-statistics.html

25. Arber DA, Orazi A, Hasserjian R, Thiele J, Borowitz MJ, Le Beau MM, et al. The 2016 revision to the World Health Organization classification of myeloid neoplasms and acute leukemia. Blood (2016) 127(20):2391-405. doi:10.1182/blood-2016-03-643544

26. Arber DA, Borowitz MJ, Cessna M, Etzell J, Foucar K, Hasserjian RP, et al. Initial diagnostic workup of acute leukemia: guideline from the college of American pathologists and the American society of hematology. Arch Pathol Lab Med (2017) 141(10):1342-93. doi:10.5858/arpa.2016-0504-CP

27. Iacobucci I, Mullighan CG. Genetic basis of acute lymphoblastic leukemia. J Clin Oncol (2017) 35(9):975-83. doi:10.1200/JCO.2016.70.7836

28. Roberts KG, Pei D, Campana D, Payne-Turner D, Li Y, Cheng C, et al. Outcomes of children with BCR-ABL1-like acute lymphoblastic leukemia treated with risk-directed therapy based on the levels of minimal residual disease. JClin Oncol (2014) 32(27):3012-20. doi:10.1200/JCO.2014. 55.4105

29. Hunger SP, Mullighan CG. Redefining ALL classification: toward detecting high-risk ALL and implementing precision medicine. Blood (2015) 125(26):3977-87. doi:10.1182/blood-2015-02-580043

30. Reshmi SC, Harvey RC, Roberts KG, Stonerock E, Smith A, Jenkins H, et al. Targetable kinase gene fusions in high-risk B-ALL: a study from the Children's Oncology Group. Blood (2017) 129(25):3352-61. doi:10.1182/ blood-2016-12-758979

31. Roberts KG, Li Y, Payne-Turner D, Harvey RC, Yang Y-L, Pei D, et al. Targetable kinase-activating lesions in Ph-like acute lymphoblastic leukemia. N Engl J Med (2014) 371(11):1005-15. doi:10.1056/NEJMoa1403088

32. SchinnerlD, Fortschegger K, Kauer M, Marchante JRM, Kofler R, Den Boer ML, et al. The role of the Janus-faced transcription factor PAX5-JAK2 in acute lyphoblastic leukemia. Blood (2015) 125(8):1282-91. doi:10.1182/blood-201404-570960

33. Nebral K, Denk D, Attarbaschi A, König M, Mann G, Haas OA, et al. Incidence and diversity of PAX5 fusion genes in childhood acute lymphoblastic leukemia. Leukemia (2009) 23(1):134-43. doi:10.1038/leu. 2008.306
34. Harrison CJ. Blood Spotlight on iAMP21 acute lymphoblastic leukemia (ALL), a high-risk pediatric disease. Blood (2015) 125(9):1383-6. doi:10.1182/ blood-2014-08-569228

35. Moorman AV, Robinson H, Schwab C, Richards SM, Hancock J, Mitchell CD, et al. Risk-directed treatment intensification significantly reduces the risk of relapse among children and adolescents with acute lymphoblastic leukemia and intrachromosomal amplification of chromosome 21: a comparison of the MRC ALL97/99 and UKALL2003 trials. J Clin Oncol (2013) 31(27):3389-96. doi:10.1200/JCO.2013.48.9377

36. Heerema NA, Carroll AJ, Devidas M, Loh ML, Borowitz MJ, Gastier-Foster JM, et al. Intrachromosomal amplification of chromosome 21 is associated with inferior outcomes in children with acute lymphoblastic leukemia treated in contemporary standard-risk Children's Oncology Group studies: a report from the Children's Oncology Group. J Clin Oncol (2013) 31(27):3397-402. doi:10.1200/JCO.2013.49.1308

37. Radtke I, Mullighan CG, Ishii M, Su X, Cheng J, Ma J, et al. Genomic analysis reveals few genetic alterations in pediatric acute myeloid leukemia. Proc Natl Acad Sci U S A (2009) 106(31):12944-9. doi:10.1073/pnas. 0903142106

38. De Kouchkovsky I, Abdul-Hay M. Acute myeloid leukemia: a comprehensive review and 2016 update. Blood Cancer J (2016) 6(7):e441. doi:10.1038/ bcj. 2016.50

39. Jaffe ES. Pathology and Genetics of Tumours of Haematopoietic and Lymphoid Tissues. Lyon, France: IARC (2001). 351 p.

40. Vardiman JW, Thiele J, Arber DA, Brunning RD, Borowitz MJ, Porwit A, et al. The 2008 revision of the World Health Organization (WHO) classification of myeloid neoplasms and acute leukemia: rationale and important changes. Blood (2009) 114(5):937-51. doi:10.1182/blood-2009-03-209262

41. Liang D-C, Shih L-Y, Hung I-J, Yang C-P, Chen S-H, Jaing T-H, et al. FLT3TKD mutation in childhood acute myeloid leukemia. Leukemia (2003) 17(5): 883-6. doi:10.1038/sj.leu.2402928

42. Ghosh K, Swaminathan S, Madkaikar M, Gupta M, Kerketta L, Vundinti B. FLT3 and NPM1 mutations in a cohort of AML patients and detection of a novel mutation in tyrosine kinase domain of FLT3 gene from Western India. Ann Hematol (2012) 91(11):1703-12. doi:10.1007/s00277-012$1509-\mathrm{z}$

43. Badar T, Patel KP, Thompson PA, DiNardo C, Takahashi K, Cabrero M, et al. Detectable FLT3-ITD or RAS mutation at the time of transformation from MDS to AML predicts for very poor outcomes. Leuk Res (2015) 39(12): 1367-74. doi:10.1016/j.leukres.2015.10.005

44. Stone RM, Fischer T, Paquette R, Schiller G, Schiffer CA, Ehninger G, et al. Phase IB study of the FLT3 kinase inhibitor midostaurin with chemotherapy in younger newly diagnosed adult patients with acute myeloid leukemia. Leukemia (2012) 26(9):2061-8. doi:10.1038/leu.2012.115

45. Dvorak CC, Loh ML. Juvenile myelomonocytic leukemia: molecular pathogenesis informs current approaches to therapy and hematopoietic cell transplantation. Front Pediatr (2014) 2:25. doi:10.3389/fped.2014. 00025

46. Obenauer JC, Kavelaars FG, Sanders MA, de Vries ACH, de Haas V, Berna Beverloo H, et al. Recurrently affected genes in juvenile myelomonocytic leukaemia. Br J Haematol (2017). doi:10.1111/bjh.14737

47. Stieglitz E, Taylor-Weiner AN, Chang TY, Gelston LC, Wang Y-D, Mazor T, et al. Corrigendum: the genomic landscape of juvenile myelomonocytic leukemia. Nat Genet (2016) 48(1):101. doi:10.1038/ng0116-101a

48. The Global Alliance for Genomics and Health. A federated ecosystem for sharing genomic, clinical data. Science (2016) 352(6291):1278-80. doi:10.1126/ science.aaf6162

49. Marx V. The DNA of a nation. Nature (2015) 524(7566):503-5. doi:10.1038/ 524503a

50. Collins FS, Varmus H. A new initiative on precision medicine. N Engl J Med (2015) 372(9):793-5. doi:10.1056/NEJMp1500523

51. Gerstung M, Papaemmanuil E, Martincorena I, Bullinger L, Gaidzik VI, Paschka P, et al. Precision oncology for acute myeloid leukemia using a knowledge bank approach. Nat Genet (2017) 49(3):332-40. doi:10.1038/ ng.3756

52. Rehm HL, Bale SJ, Bayrak-Toydemir P, Berg JS, Brown KK, Deignan JL, et al. ACMG clinical laboratory standards for next-generation sequencing. Genet Med (2013) 15(9):733-47. doi:10.1038/gim.2013.92 
53. Belkadi A, Bolze A, Itan Y, Cobat A, Vincent QB, Antipenko A, et al. Whole-genome sequencing is more powerful than whole-exome sequencing for detecting exome variants. Proc Natl Acad Sci U S A (2015) 112(17):5473-8. doi:10.1073/pnas.1418631112

54. Shivarov V, Bullinger L. Expression profiling of leukemia patients: key lessons and future directions. Exp Hematol (2014) 42(8):651-60. doi:10.1016/j. exphem.2014.04.006

55. Shen T, Pajaro-Van de Stadt SH, Yeat NC, Lin JC-H. Clinical applications of next generation sequencing in cancer: from panels, to exomes, to genomes. Front Genet (2015) 6:215. doi:10.3389/fgene.2015.00215

56. Flusberg BA, Webster DR, Lee JH, Travers KJ, Olivares EC, Clark TA, et al. Direct detection of DNA methylation during single-molecule, real-time sequencing. Nat Methods (2010) 7(6):461-5. doi:10.1038/nmeth.1459

57. Rhoads A, Au KF. PacBio sequencing and its applications. Genomics Proteomics Bioinformatics (2015) 13(5):278-89. doi:10.1016/j.gpb.2015.08.002

58. Simpson JT, Workman RE, Zuzarte PC, David M, Dursi LJ, Timp W. Detecting DNA cytosine methylation using nanopore sequencing. Nat Methods (2017) 14(4):407-10. doi:10.1038/nmeth.4184
59. Loose MW. The potential impact of nanopore sequencing on human genetics. Hum Mol Genet (2017) 26(R2):R202-7. doi:10.1093/hmg/ddx287

60. Zheng GXY, Lau BT, Schnall-Levin M, Jarosz M, Bell JM, Hindson CM, et al. Haplotyping germline and cancer genomes with high-throughput linked-read sequencing. Nat Biotechnol (2016) 34(3):303-11. doi:10.1038/ nbt. 3432

Conflict of Interest Statement: The authors declare that the research was conducted in the absence of any commercial or financial relationships that could be construed as a potential conflict of interest.

Copyright (c) 2017 Lalonde, Wertheim and Li. This is an open-access article distributed under the terms of the Creative Commons Attribution License (CC BY). The use, distribution or reproduction in other forums is permitted, provided the original author(s) or licensor are credited and that the original publication in this journal is cited, in accordance with accepted academic practice. No use, distribution or reproduction is permitted which does not comply with these terms. 